\title{
Recurrence Following Pulmonary Hydatid Disease Surgery
}

\author{
Mohsen Sokouti · Samad E. J. Golzari · \\ Servet Kayhan · Babak Sabermarouf
}

Published online: 10 July 2013

(c) Société Internationale de Chirurgie 2013

\section{Dear Editor,}

Hydatid disease is still a major health concern in the World Health Organization's Eastern Mediterranean Region countries [1]. The disease has the ability to affect all organs, but the liver is the organ most commonly affected followed by the lungs. Numerous modalities have been introduced for diagnosing pulmonary hydatid disease [2]. Surgery, however, remains the treatment of choice, with various approaches used to save as much of the lungs as possible. Recurrences appear because of intraoperative spillage of the hydatid daughter cysts into the pleural cavity, with most studies indicating recurrence rates for pulmonary hydatid disease of 4.6-22.0\% [3-5].

Mahmodlou et al. [1], despite a cyst perforation rate of $29.4 \%$, reported no recurrences. They compared their results with the study of Mottaghian and Saidi [5] in which a recurrence rate of $1.5-12.0 \%$ had been reported. The

\section{Sokouti}

Department of Cardiothoracic Surgery, Tabriz University of

Medical Sciences, Tabriz, Iran

\section{S. E. J. Golzari ( $\square)$}

Cardiovascular Research Center, Tabriz University of Medical

Sciences, Tabriz, Iran

e-mail:dr.golzari@hotmail.com

\section{S. E. J. Golzari}

Students' Research Committee, Tabriz University of Medical

Sciences, Tabriz, Iran

\section{S. Kayhan}

Department of Pulmonary Medicine, Recep Tayyip Erdogan University, Rize, Turkey

B. Sabermarouf

Neurosciences Research Center (NSRC), Tabriz University of Medical Sciences, Tabriz, Iran follow-up period of the studies conducted by Mottaghian and Saidi [3-5] was 6 months to 3 years, a range reported by most authors to be sufficient for identifying recurrence of the disease, although some use a minimum of 4 years for their follow-up protocol. The follow-up period of the study conducted by Mahmodlou et al. was 6 months, a time span too short to compare their results with those of similar studies or to make any definitive statements about a zero recurrence rate.

Accidental rupture or spillage of cysts during the operation might lead to consequent regrowth of the cysts. Thus, the operative field should be kept clear of contamination by irrigating the pleural cavity with hypertonic saline. Also, the cysts should be handled with very gentle manipulation. These precautionary measures along with chemotherapy and a thorough follow-up program might avoid recurrence of the disease.

\section{References}

1. Mahmodlou R, Sepehrvand N, Nasiri M (2013) Saucerization: a modified uncapitonnage method of surgery for pulmonary hydatidosis. World J Surg. doi:10.1007/s00268-013-2093-7

2. Golzari SE, Sokouti M, Ghaffari A et al (2013) Ultrasonography in diagnosis of pulmonary hydatid cysts. Lancet Infect Dis 13:294

3. Little JM, Hollands MJ, Ekberg H (1988) Recurrence of hydatid disease. World J Surg 12:700-704

4. Prousalidis J, Kosmidis C, Anthimidis G et al (2012) Postoperative recurrence of cystic hydatidosis. Can J Surg 55:15-20

5. Mottaghian H, Saidi F (1978) Postoperative recurrence of hydatid disease. Br J Surg 65:237-242 